\title{
Cytoreductive surgery and hyperthermic intraperitoneal chemotherapy for sarcomatosis from uterine adenosarcoma
}

\author{
Michael P. O'Leary' ${ }^{1}$, Sinziana Dumitra' ${ }^{1}$, Bryan Goldner' ${ }^{1}$, \\ Mark Wakabayashi², Byrne Lee ${ }^{1 *}$
}

Department of Surgery, Division of Surgical Oncology, Division of Gynecologic Oncology, City of Hope National Medical Center, Duarte, CA, USA

Received: 30 March 2017

Accepted: 27 April 2017

*Correspondence:

Dr. Byrne Lee,

E-mail: bylee@coh.org

Copyright: (c) the author(s), publisher and licensee Medip Academy. This is an open-access article distributed under the terms of the Creative Commons Attribution Non-Commercial License, which permits unrestricted non-commercial use, distribution, and reproduction in any medium, provided the original work is properly cited.

\begin{abstract}
Uterine adenosarcoma has poor prognosis and management of this disease is controversial. We describe a case of sarcomatosis secondary to recurrent uterine adenosarcoma who underwent cytoreductive surgery (CS) and hyperthermic intraoperative peritoneal chemotherapy (HIPEC). A 52 year-old female presented with perimenopausal menometrorrhagia. She underwent laparoscopic hysterectomy and bilateral salpingo-oopherectomy with pathology showing uterine adenosarcoma. She developed a pelvic recurrence 2 years later. A pelvic exenteration was then performed and within 8 months, she recurred. CS/HIPEC with Cisplatin was performed. Six weeks post-operatively, the patient was found to have recurrence again. This case describes the use of CS/HIPEC as a treatment modality for uterine adenosarcoma with sarcomatosis. Despite CS and HIPEC, the patient developed an aggressive recurrence within six weeks of her surgery date. We recommend a multidisciplinary approach to this disease with the recognition that CS/HIPEC may offer little benefit as a salvage therapy based on this case.
\end{abstract}

Keywords: Cytoreduction, Hyperthermic intraperitoneal chemotherapy, Uterine sarcoma

\section{INTRODUCTION}

Uterine sarcomas are rare, accounting for only $1 \%$ of all gynecologic cancers and 3 to $7 \%$ of all uterine cancers. ${ }^{1}$ The uterine adenosarcoma subtype occurs in less than $5 \%$ of uterine sarcomas. The poor overall survival and aggressive nature of the disease has been well documented. ${ }^{2-4}$ Recurrence rates as high as 70 percent with traditional treatment modalities have forced practitioners to rethink treatment options in effort to improve both disease free and overall survival. ${ }^{5}$

Cytoreductive surgery (CS) and hyperthermic intraoperative peritoneal chemotherapy (HIPEC) are established treatment modalities for gynecologic malignancies. The combination therapy for recurrent ovarian cancer confers a doubling of overall survival when compared to CS alone. ${ }^{6}$ Large-scale studies of HIPEC and CS for uterine sarcoma are challenging given the rarity of the disease process, therefore outcomes are inferred from small case series. ${ }^{7}$

The purpose of this report is to augment the existing literature describing the utilization of CS and HIPEC for uterine adenosarcoma. This is only the second case report of a patient receiving this treatment modality for uterine adenosarcoma.

\section{CASE REPORT}

A retrospective review of our institutional cytoreduction database from 2009 to 2015 was performed following 
approval by the institution review board. A total of 157 patients were evaluated for CS and HIPEC with 115 (73.2\%) undergoing CS and HIPEC. One patient was found to have peritoneal sarcomatosis from a recurrent uterine adenosarcoma and underwent CS and HIPEC. Computed tomography (CT) of the abdomen and pelvis were obtained prior to all surgical interventions. The histopathology was reviewed for each procedure. A review of the literature was performed to gather information on epidemiology, natural history, and management of the disease.

A 52-year-old Korean-American female presented with perimenopausal menometrorrhagia. She had no past medical or surgical history and took no medications. Her social history for tobacco, drug and alcohol use were negative. Her family history consisted of pancreatic cancer in her father and paternal uncle who died at ages 52 and 62 years old, respectively. A broad workup for genetic predisposition for cancers was negative for known genetic mutations. Her initial evaluation revealed a polypoid mass protruding from the cervical os and she underwent laparoscopic hysterectomy with bilateral salpingo-oophorectomy prior to referral to our institution.

Pathology revealed a $5 \times 5.5 \mathrm{~cm}$, stage IIA uterine adenosarcoma with spread to the right ovary. Sarcomatous overgrowth, defined as sarcoma occupying at least $25 \%$ of the tumor, was present (Figure 1a and 1b). She was referred to our institution and then received 45 Gray of pelvic radiation.

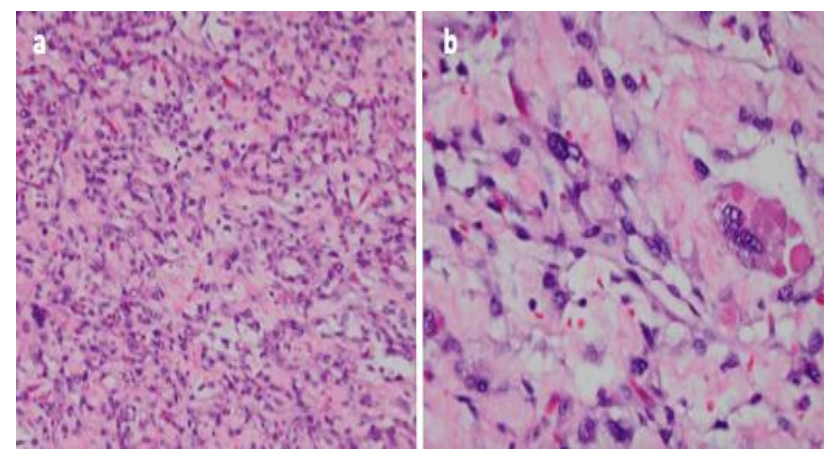

Figure 1: Histology showing uterine adenosarcoma at (a) $100 \mathrm{X}$ and (b) $400 \mathrm{X}$ with $>95 \%$ sarcomatous overgrowth.

At 24 months surveillance, CT of the abdomen and pelvis revealed a right anterior abdominal wall recurrence for which she underwent primary resection, which was then followed by adjuvant gemcitabine and docetaxel (Figure $2 \mathrm{a}$ and $2 \mathrm{~b}$ ). She completed three rounds of chemotherapy but did not complete the full course secondary to severe fatigue.

At 32 months from her initial surgery, she presented with abdominal pain. A CT revealed a pelvic recurrence of her disease (Figure 2c). She then underwent total pelvic exenteration, with creation of an ileal conduit and end sigmoid colostomy. Eight months following this procedure, surveillance $\mathrm{CT}$ scan again revealed recurrent disease at the right anterior abdominal wall (Figure 2d).
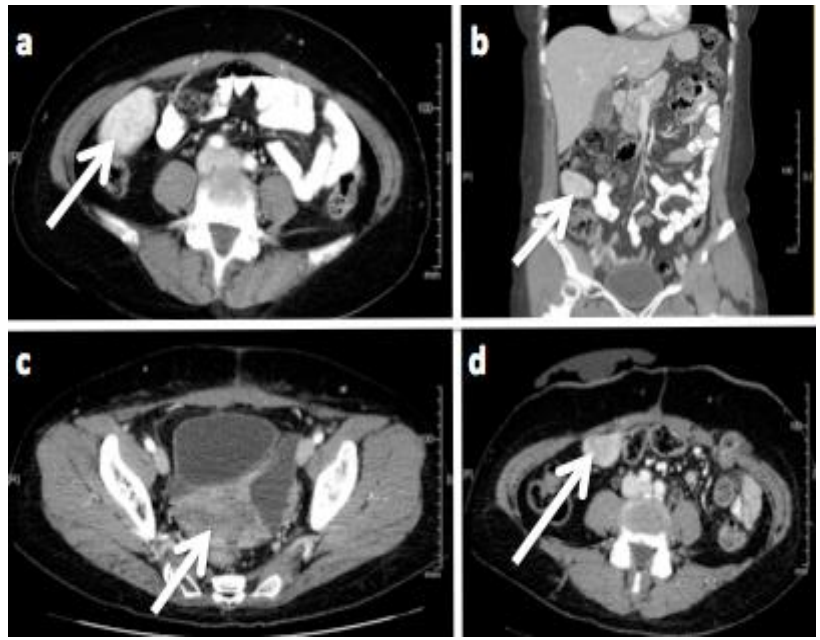

Figure 2: Surveillance computed tomography with solid arrow points to all locations of recurrent uterine adenosarcoma with time in reference to primary surgery: (a) and (b) 24 months $-6 \times 4 \times 3 \mathrm{~cm}$ peritoneal recurrence, (c) 32 months - complex pelvic mass (d) 40 months - peritoneal recurrence at right anterior abdominal wall.

An interdisciplinary tumor board recommended CS and HIPEC. The patient underwent a CS and HIPEC procedure with intraoperative findings of a peritoneal cancer index score of 9. A hemorrhagic mass was seen adherent to the right anterior abdominal wall (Figure 3) and was resected en-bloc. Her completion of cytoreduction score was 0. HIPEC was performed using Cisplatin infused for 60 minutes at 41 degrees Celsius.

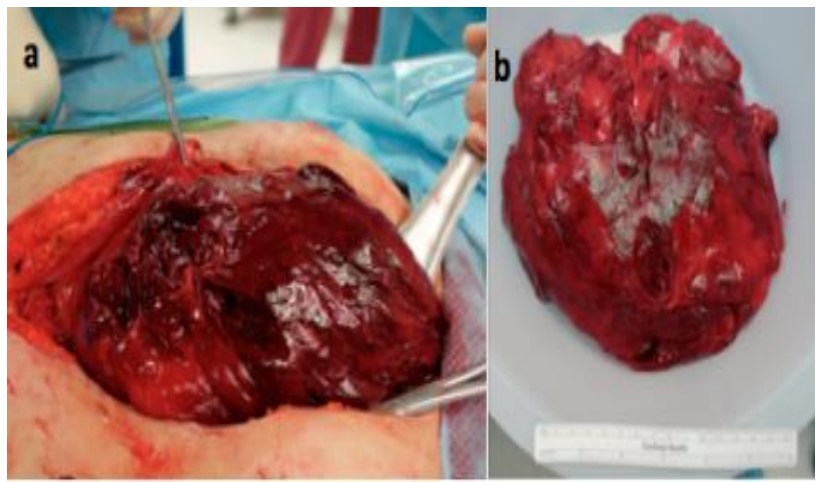

Figure 3: Intraoperative pictures of (a) protruding hemorrhagic recurrent uterine adenosarcoma and (b) pathological specimen following resection.

Six weeks following CS and HIPEC, she presented with complaints of vaginal discharge. She was found to be tachycardic and hypotensive. Admission CT scan showed a complex multiloculated cystic mass, which was consistent with a recurrent uterine adenosarcoma. After a 
multidisciplinary tumor board discussion as well as discussion with the patient and her family, she opted for palliative care and hospice. She expired three months later.

\section{DISCUSSION}

Uterine sarcoma is typically divided into three histopathological categories: leiomyosarcoma, carcinosarcoma and adenosarcoma. ${ }^{1,8}$ Uterine leiomyosarcoma is the most common (40\%) while adenosarcoma only occurs in $5 \%$ of uterine sarcomas. ${ }^{5}$ The adenosarcoma subtype typically portends good prognosis when compared to other histologic subtypes of uterine sarcoma; however, a quarter of these patients will ultimately die from their disease. ${ }^{1,9}$

Stage, depth of myometrial invasion, mitotic index, grade, and presence of sarcomatous overgrowth have been evaluated as prognostic factors. ${ }^{1,3,9-11}$ Carroll et al found progression free survival, disease free survival, and overall survival were significantly worse in patients with sarcomatous overgrowth. Moreover, they showed sarcomatous overgrowth to be associated with more advanced disease. ${ }^{3}$ This is consistent with other studies whereby 5-year overall survival is similar between adenosarcoma with sarcomatous overgrowth to carcinosarcoma at $22 \% .{ }^{12}$ Extrauterine disease has been found to have varying incidence between reports. The incidence has been reported between 2 to $8 \%$ in some series, but in the presence of sarcomatous overgrowth can be as high as $70 \% .^{3,5,9,12}$ On review of the histology, our patient had at least 90 percent sarcomatous overgrowth.

Current treatment modalities based on the National Comprehensive Cancer Network (NCCN) for uterine carcinoma recommend primary total abdominal hysterectomy with bilateral salpingo-oopherectomy. However, no consensus has been reached on adjuvant chemotherapy or radiation therapy given the rarity of the disease. ${ }^{13}$ Recurrence is common, occurring in up to 77 percent of cases with sarcomatous overgrowth. Guidelines cannot be made regarding the benefit of secondary cytoreduction after disease recurrence since only observational studies have been performed, and no statistically significant, improved progression free survival has been published. ${ }^{3,14}$

CS and HIPEC have been utilized for peritoneal sarcomatosis secondary to gastrointestinal sarcomas, liposarcoma and uterine sarcomas, excluding adenosarcoma, with overall results similar to conventional therapies. ${ }^{15}$ Only one case has been reported using CS and HIPEC for uterine adenosarcoma. This patient developed 4 separate recurrences requiring CS and HIPEC at each surgery, but was alive at 55 months post diagnosis. ${ }^{7}$ Our patient similarly developed recurrent disease but the discussion of CS and HIPEC was only performed at her last surgery. Overall, her survival from time of diagnosis was 43 months.
The patient developed diffuse intraperitoneal disease recurrence and required admission only 6 weeks following CS and HIPEC, despite a completion of cytoreduction score of 0 . Numerous reasons for disease progression can be proposed. Histologically, the tumor at the time of CS and HIPEC appeared similar to the histopathology at time of diagnosis, so it is unlikely that a different tumor biology caused the hasty progression of her cancer following CS and HIPEC. Also unlikely are surgical or mechanical components of CS and HIPEC. This is evident by a 55 month survival after 4 separate CS and HIPEC procedures in the other published case. ${ }^{7}$

Uterine adenosarcoma, especially with sarcomatous overgrowth, portends a poor prognosis with current treatment recommendations. CS and HIPEC should be considered as a viable treatment modality. However, patients must be aware survival benefit, or lack thereof, cannot be established by the existing literature given the rare nature of the disease and treatment. If HIPEC and CS are attempted, they should be implemented early in the disease course as they can have significant morbidity as a salvage therapy. Large studies to investigate outcomes of uterine adenosarcoma and CS with HIPEC will be challenging and multidisciplinary teams should be utilized to care for patients with this challenging disease process.

We describe the second case of CS and HIPEC for sarcomatosis secondary to uterine adenosarcoma. Given the rare nature of the disease, large prospective outcome based guidelines will be difficult to establish. A multidisciplinary approach should evaluate the benefit of CRS and HIPEC early in the disease course as it may offer little benefit as a salvage therapy.

Funding: No funding sources

Conflict of interest: None declared

Ethical approval: Not required

\section{REFERENCES}

1. D'Angelo E, Prat J. Uterine sarcomas: a review. Gynecol Oncol. 2010;116:131-9.

2. Major FJ, Blessing JA, Silverberg SG, Morrow CP, Creasman WT, Currie JL et al. Prognostic factors in early-stage uterine sarcoma. A Gynecologic Oncology Group study. Cancer. 1993;71:1702-9.

3. Carroll A, Ramirez PT, Westin SN, Soliman PT, Munsell MF, Nick AM et al. Uterine adenosarcoma: an analysis on management, outcomes, and risk factors for recurrence. Gynecol Oncol. 2014;135:455-61.

4. Giuntoli RL, Metzinger DS, DiMarco CS, Cha SS, Sloan JA, Keeney GL et al. Retrospective review of 208 patients with leiomyosarcoma of the uterus: prognostic indicators, surgical management, and adjuvant therapy. Gynecol Oncol. 2003;89:460-9.

5. Abeler VM, Røyne O, Thoresen S, Danielsen HE, Nesland JM, Kristensen GB. Uterine sarcomas in 
Norway. A histopathological and prognostic survey of a total population from 1970 to 2000 including 419 patients. Histopathol. 2009;54:355-64.

6. Spiliotis J, Halkia E, Lianos E, Kalantzi N, Grivas A, Efstathiou E et al. Cytoreductive surgery and HIPEC in recurrent epithelial ovarian cancer: a prospective randomized phase III study. Ann Surg Oncol. 2015;22:1570-5.

7. Jimenez WA, Sardi A, Nieroda C, Gushchin V. Cytoreductive surgery and hyperthermic intraperitoneal chemotherapy in the management of recurrent high-grade uterine sarcoma with peritoneal dissemination. Am J Obstet Gynecol. 2014;210:259.e1-8.

8. Prat J. FIGO staging for uterine sarcomas. Int J Gynaecol Obstet Off Organ Int Fed Gynaecol Obstet. 2009;104:177-8.

9. Clement PB, Scully RE. Mullerian adenosarcoma of the uterus: a clinicopathologic analysis of 100 cases with a review of the literature. Hum Pathol. 1990;21:363-81.

10. Friedlander ML, Covens A, Glasspool RM, Hilpert F, Kristensen G, Kwon S et al. Gynecologic Cancer InterGroup (GCIG) consensus review for mullerian adenosarcoma of the female genital tract. Int $\mathrm{J}$ Gynecol Cancer Off J Int Gynecol Cancer Soc. 2014;24:S78-82.

11. Zaloudek CJ, Norris HJ. Adenofibroma and adenosarcoma of the uterus: a clinicopathologic study of 35 cases. Cancer. 1981;48:354-66.
12. Krivak TC, Seidman JD, McBroom JW, MacKoul PJ, Aye LM, Rose GS. Uterine adenosarcoma with sarcomatous overgrowth versus uterine carcinosarcoma: comparison of treatment and survival. Gynecol Oncol. 2001;83:89-94.

13. National Comprehensive Cancer Network. Uterine Neoplasms Version 2.2016. www.nccn.org. Accessed Feb 2016.

14. Tanner EJ, Toussaint T, Leitao MM, Hensley ML, Soslow RA, Gardner GJ et al. Management of uterine adenosarcomas with and without sarcomatous overgrowth. Gynecol Oncol. 2013;129:140-4.

15. Baratti D, Pennacchioli E, Kusamura S, Fiore M, Balestra MR, Colombo $\mathrm{C}$ et al. Peritoneal sarcomatosis: is there a subset of patients who may benefit from cytoreductive surgery and hyperthermic intraperitoneal chemotherapy? Ann Surg Oncol. 2010; $17: 3220-8$

Cite this article as: O'Leary MP, Dumitra S, Goldner B, Wakabayashi M, Lee B. Cytoreductive surgery and hyperthermic intraperitoneal chemotherapy for sarcomatosis from uterine adenosarcoma. Int J Reprod Contracept Obstet Gynecol 2017;6:2618-21. 